\title{
Studies on the Dynamics of a Supercavitating Vehicle
}

\author{
MA Fu-qiang ${ }^{1, a^{*}}$, LIU Yan-sen ${ }^{1, a}$, WANG Yang ${ }^{1, a}$ \\ ${ }^{1}$ Dalian Scientific Test \& Control Technology Institute, Dalian, China \\ adlinstitute@vip.163.com
}

Keywords: Supercavitating vehicle; Vehicle trajectory; Stability; Numerical simulation Abstract.Due to imperfect water entry, a high speed supercavitating vehicle, while moving in the forward direction, rotatesinside the cavity. This rotation leads to a series of impacts between the vehicle body and the cavity wall. The impacts affect the trajectory as well as the stability of motion of the vehicle. The present paper discusses the dynamics of such a vehicle. Equations of motion of the vehicle are developed for two distinct cases of motion. Case I: the vehicle moves in the cavity without interaction with the cavity wall, and Case II: the impacts on the cavity wall. The equations are found to be coupled and nonlinear. A simple model based on the concepts of flow planes is used to determine the forces acting on the vehicle. The results show that despite the impacts with the cavity wall, the vehicle nearly follows a straight line path. The frequency of the impacts between the vehicle tail and cavity boundary increases initially, reaches a maximum, and then decreases gradually.

\section{Introduction}

When an underwater vehicle moves at high $\operatorname{speeds}(70 \geq \mathrm{m} / \mathrm{s})$, a cavitation field that exhibits a very low drag is created. This cavitation field is called a supercavity ${ }^{[1]}$. As a result of the supercavity, the vehicle is in contact with the water only at the nose during most of its flight. The presence of the cavity changes the nature of motion of the vehicle. The vehicle while moving in the forward direction also starts rotating about its tip in the vertical plane. Because of this rotation, the tail of the vehicle impacts on the cavity wall, and then it bounces back and impacts on the opposite side of the cavity and this type of oscillatory motion continues till the diameter of the cavity becomes small. while the vehicle moves with the cavity boundary in contact with the side surface and ultimately the cavity disappears ${ }^{[2-4]}$. In the present paper, the dynamics of a supercavitating vehicle whose motion is confined to a plane is studied. Equations of motion of the vehicle are developed for two distinct cases of motion: When the vehicle moves in the cavity without interaction with the cavity boundary, and when the vehicle impacts on the cavity boundary.

\section{Analysis method and mathematic model}

\section{Dynamic model of supercavitating vehicle}

In Case I, the only force acting on the vehicle is at the nose, as shown in Fig.1(a), whereas in Case II, there are additional forces acting at the point of impact of the vehicle tail, as shown in Fig.1(b). The impact force and moment calculations are based on the method described by Milwitzky ${ }^{[5]}$. The drag force acting on the nose of the vehicle is determined from the experimental results given by Kiceniuk[6]. The formulae used to calculate the gross features of the cavity, i.e., the length and maximum diameter, are derived by Garabedian ${ }^{[7]}$. Throughout this paper, it is assumed that the vehicle has the geometry shown in Fig.2. 


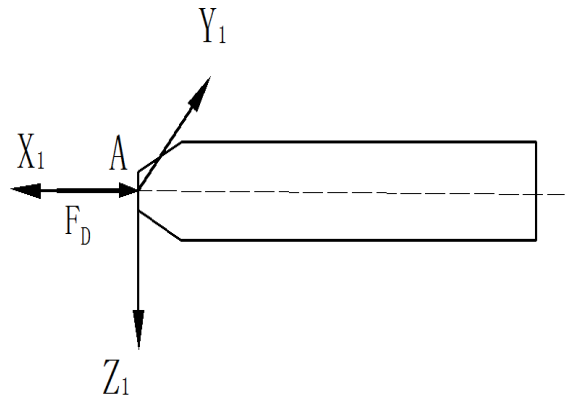

(a) Case I

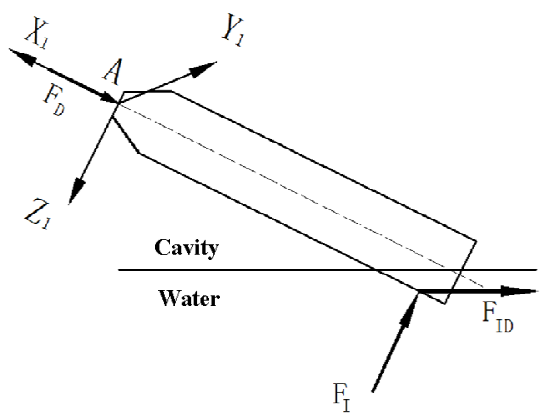

(b) Case II

Fig.1 Forces acting on the projectile for case

During cavity-running, the vehicle is in contact with the water only at the nose (neglecting impacts). The nose of the vehicle is assumed to be a disc of diameter. The lift and drag coefficients for a disc for different angles of attack have been found experimentally ${ }^{[8]}$. They are given by:

$$
C_{L}=k \sin \alpha \cos \alpha \text { and } C_{d}=k \cos \alpha \cos \alpha
$$

Then the lift and the drag forces are then given by:

$$
F_{D L}=\frac{1}{2} \rho A_{\mathrm{o}} v^{2} C_{L} \text { and } F_{D D}=\frac{1}{2} \rho A_{\mathrm{o}} v^{2} C_{\mathrm{d}}
$$

Using these expressions for the forces, the magnitude of the total force acting on the nose is given by:

$$
F_{D}=\frac{1}{2} \rho A_{0} v^{2} k \cos \alpha
$$

In terms of the components of velocity in the $x_{1}$ and $z_{1}$ directions, the magnitude of the force is given by:

$$
F_{D}=\frac{1}{2} \rho A_{o} k\left(U^{2}+W^{2}\right) \frac{U}{\sqrt{\left(U^{2}+W^{2}\right)}}
$$

Where, $\rho$ is the density of water; $A_{o}$ is the area of the disc; $k$ is a constant.

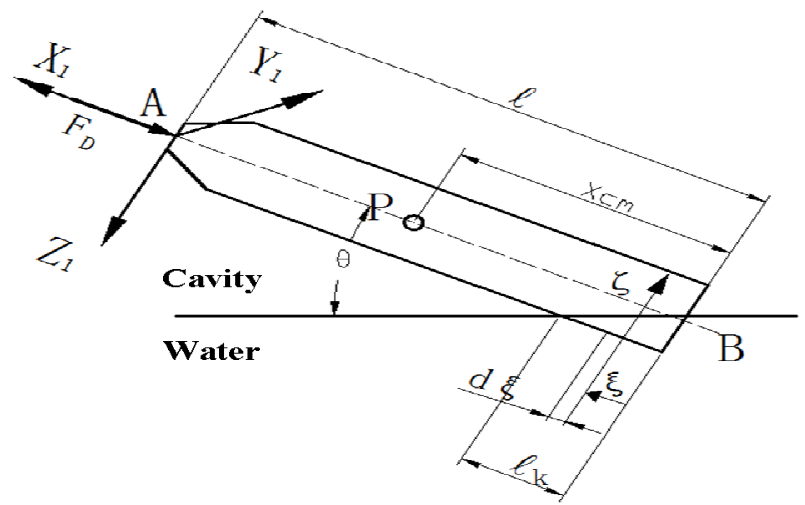

Fig 2. Culculation of force and moment

The vehicle impacts with the cavity boundary as shows in Fig2. The angle at which the vehicle impacts is defined by $\theta$. A new set of axes $(\xi, \zeta)$ is defined at point $\mathrm{B}$. The $\xi$-axis points in the same direction as the $x_{1}$-axis while the $\zeta$-axis points in the direction opposite to the $z_{1}$-axis. The wetted length of the vehicle is defined by $L_{k}$.

Normal force acting at a cross section of the vehicle which is at a distance of $\xi$ from the point B is given by:

$$
f=-\frac{d\left(m_{a p p} W_{\xi}\right)}{d t}
$$

Where: the virtual mass is $m_{a p p}=K \rho d \zeta$ and the magnitude of the velocity of the point under consideration in the $z_{1}$ direction $W_{\xi}=(W+(l-\xi) Q)$. 
we get an expression for the force exerted by a single flow plane on the vehicle. This force is then integrated over the wetted length to give the total force exerted on the vehicle. The expression for this force is given by:

$$
F_{I}=K \rho d\left(C_{1}+C_{2} w^{\alpha}+C_{3} Q\right) \text { and } M_{I}=K \rho d\left(E_{1}+E_{2} x^{\alpha}+E_{3} \phi\right)
$$

To find the force and moment exerted by the water on the vehicle at any instant it is necessary to know the wetted length at that instant. The numerical procedure for calculating the wetted length is given below ${ }^{[9]}$. Fig. 3 shows a vehicle hitting the cavity boundary at instant at $t=t_{0}$. In time interval $d t$, the point $\mathrm{B}$ travels a distance. The angle that the vehicle makes with the cavity boundary at this instant $t_{1}=t_{0}+d t$ is given by $\alpha_{1}=\alpha_{0}+Q_{0} d t$. Using this information, the depth of penetration of point $\mathrm{B}$, measured perpendicular to the vehicle axis can be calculated.

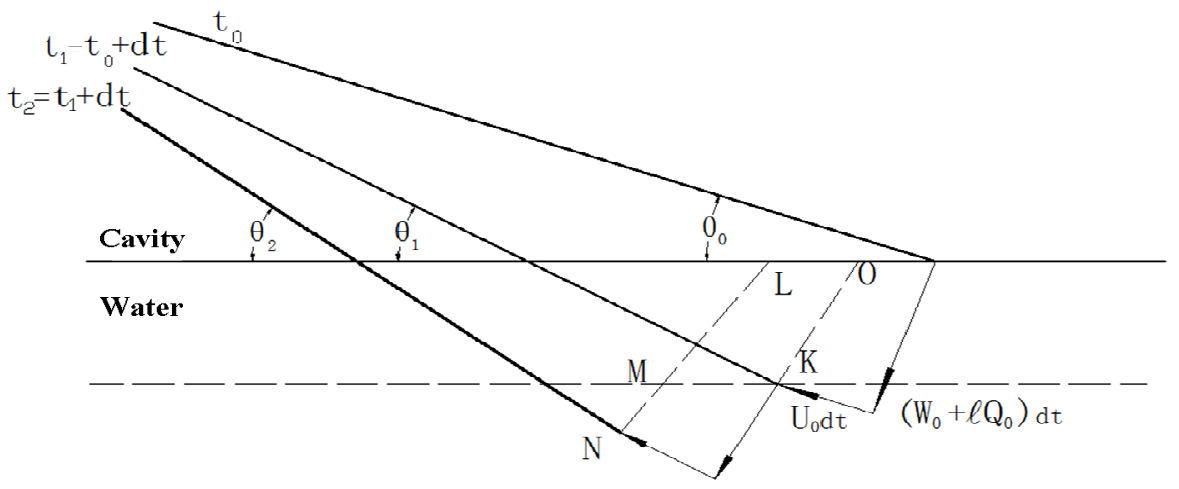

Fig. 3 Wetted length calculation

For a later instant, the change in the depth of penetration of point B is calculated similarly. For example, for the second time step, the depth of penetration of point $\mathrm{B}, l(L N)$, is given by

$$
l(L N)=l(L M)+L(M N)
$$

This procedure is continued till the depth of penetration equals zero.

\section{Kinematic model of supercavitating vehicle}

The motion of the vehicle in both phases is governed by the following three scalar equations:

$$
\begin{aligned}
& m^{i} U_{c}=\Sigma F_{X 1} \\
& m^{i} W_{c}^{\alpha}=\Sigma F_{Z 1} \\
& I^{i} \oint_{c}^{\alpha}=\Sigma M_{Y 1}
\end{aligned}
$$

The accelerations in the X1-direction and Z1-direction are:

$$
\begin{aligned}
& { }^{i} U_{c}={ }^{r} U \&_{+} Q W+\left(l-x_{c m}\right) Q^{2} \\
& { }^{i} W_{c}={ }^{r} V^{\mathcal{Q}}-Q U+\left(l-x_{c m}\right)^{i} Q_{c}^{\mathcal{Q}}
\end{aligned}
$$

where $\mathrm{m}$ is the mass of the vehicle and $I$ is the moment of inertia of the vehicle about an axis parallel to the Y1-axis and passing through its center of mass; $U_{C}$ and $Z_{C}$ are the velocity components of the center of mass of the vehicle in X1 and Z1-directions; $\Sigma F_{X 1}$ and $\Sigma F_{Z 1}$ are the net forces acting on the vehicle in the $\mathrm{X} 1$-and $\mathrm{Z1}$-directions; $\mathrm{Q}$ is the angular velocity of the vehicle; ${ }^{r} U^{\&}$ and ${ }^{r} \mathrm{~W}$ are the rate of change of $\mathrm{U}$ and $\mathrm{W}$.

The equations of motion (8)-(10), with the forces substituted, look very complicated. Although, the full equations are used for numerical simulations presented later in this paper, a simplified version of the equations is given below. These simplifed equations show the dominant terms and interdependencies.

Case I: For $U^{2} \square W^{2}, \rho A_{o} k U^{2} \square 2 m l Q^{2}, l_{k} / l \square 1$ the equations of motion can be written as: 


$$
\left\{\begin{array}{l}
U \&=\frac{F_{p}}{m}-\frac{\rho A_{o} k U^{2}}{2 m} \\
W \&=Q U \\
Q=0
\end{array}\right.
$$

Case II: For $U^{2} \square W^{2}, \rho A_{o} k U^{2} \square 2 m l Q^{2}, l_{k} / l \square 1$ the equations of motion can be written as:

$$
\begin{aligned}
& \left\{\begin{array}{l}
\dot{\&}=\frac{F_{p}}{m}-\frac{\rho A_{o} k U^{2} f\left(A_{o}, d, l, \theta\right)}{2 m} \\
W \&=W^{2}\left[\left(M_{1} l_{k}\right)+M_{2}\left(l_{k} x_{c m}\right)\left(-x_{c m}\right)\right]+2 W\left[Q M_{2} l\left(x_{c m} l_{k}\right)\left(l-x_{c m}\right)\right]+Q U \\
Q=-M_{2}\left[W^{2}\left(l_{k} x_{c m}\right)+2 W Q l\left(x_{c m} l_{k}\right)\right]
\end{array}\right. \\
& f\left(A_{o}, d, l, \theta\right)=A_{o}+\frac{d^{2}}{4} \cos ^{-1}\left(\frac{\frac{d}{2}-l_{k} \tan \theta}{\frac{d}{2}}\right)-\left(\frac{d}{2}-l_{k} \tan \theta\right) \sqrt{d l_{k} \tan \theta}
\end{aligned}
$$

\section{Numerical simulation}

In this paper ,the cavity shape is the basis of the research on the motion of the vehicle in both phases. Different cavitation morphology lead to various wetted length, wetted depth and fluid dynamics. Here, the cavity morphology is assumed to be an ellipse (actually, it is an ellipsoid in 3D). The shape and size of the elliptic cavity are characterized by its maximum diameter and its length (see Fig. 4). It is assumed that the cavity axis remains straight and always lies along the direction of the absolute velocity of the vehicle nose. The geometry of the cavity is given by:

$$
\frac{\left(x-\frac{L_{\max }}{2}\right)^{2}}{\left(\frac{L_{\max }}{2}\right)^{2}}+\frac{r_{c}(x)^{2}}{\left(\frac{d_{\max }}{2}\right)^{2}}=1
$$

and the maximum diameter and length are given by:

$$
d_{\max }=d_{c} \sqrt{\frac{C_{D}(\sigma, 0)}{\sigma}} \text { and } L_{\max }=\frac{d_{c}}{\sigma} \sqrt{C_{P}(\sigma, 0) \ln \left(\frac{1}{\sigma}\right)}
$$

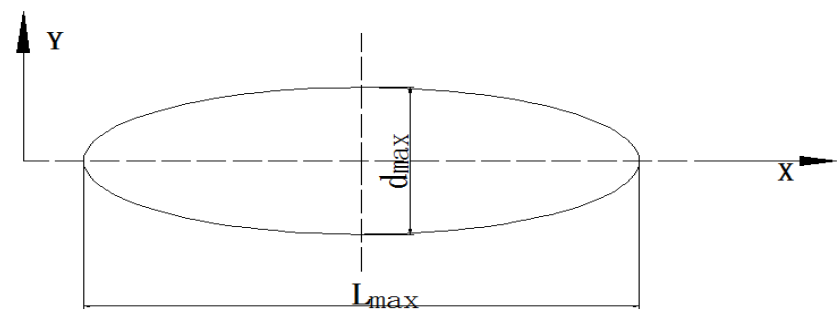

Fig. 4 Shape of the cavity

The numerical simulations are carried out for three different conditions of the vehicle. It is assumed that for every condition, the vehicle has the same mass and the same diameter. The following initial conditions are used:

Table 1 numerical condition

\begin{tabular}{ccc}
\hline Numerical condition & Initial velocity $(\mathrm{m} / \mathrm{s})$ & propelling force $(\mathrm{kn})$ \\
\hline Case I & 120 & 750 \\
Case II & 100 & 400 \\
Case III & 80 & 150 \\
\hline
\end{tabular}

\section{Results and discussion}

The results of the impact forces obtained for different conditions are shown in figs.5 (a), (b), 
(c), respectively. It seen from the figures that the magnitude of the impact forces decreases gradually during the impact. Also, as seen from the figure, the magnitude of the impact forces increases with the rise of the sailing velocity for the three different conditions.
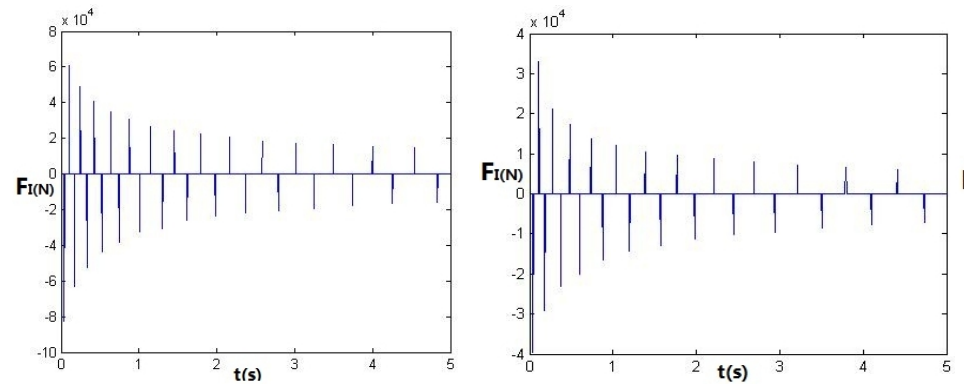

(a) Case I

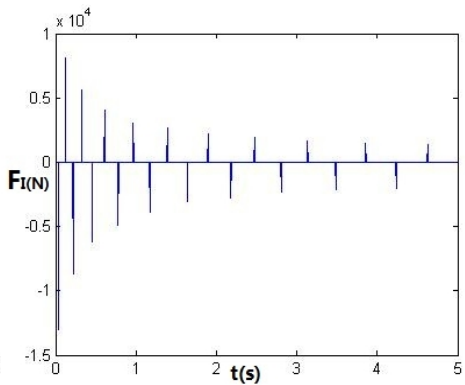

(c) Case III

Fig.5 Time evolution of the impact force

The results obtained for angular velocity are shown in Figs. 6 (a), (b), (c), respectively. It seen from the figures that the magnitude of the angular velocity decreases gradually with time evolution. Also, as seen from the figure, the magnitude of the angular velocity increases with the rise of the sailing velocity for the three different conditions. It is seen that the frequency of these impct increases and then remains nearly constant.
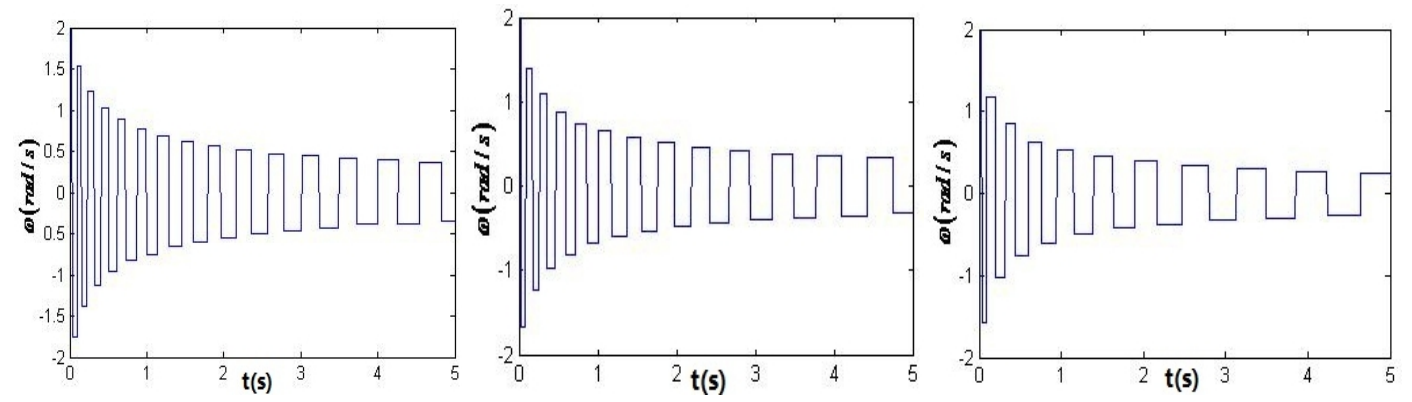

(a) Case I

(b) Case II

(c) Case III

Fig. 6 Time evolution of the angular velocity

\section{Conclusions}

A simple model for the dynamics of a high speed supercavitating vehicle has been presented in this paper. The predicted motion involves a series of impacts of the vehicle's tail with the cavity boundary. It is seen that the frequency of these impacts increases and then remains nearly constant. This is caused by the nature of variation of the angular velocity with time. It is observed that the vehicle nearly follows a straight line path, an e.ect caused due to the impacts between the vehicle tail and the cavity boundary. The dynamics of the vehicle is also found to be influenced by its mass distribution. Distributing the mass of the vehicle over a larger length increases the moment of inertia of the vehicle as well as reduces the angle of impact between the vehicle tail and the cavity boundary. Because of this, the initial rate of increase of the angular velocity and the magnitude of the maximum angular velocity reached are reduced. This leads to a reduction in the frequency of impacts, a reduction in the magnitude of the forces at the tail during impact and, consequently, a reduction in the deviation from the straight line path. 


\section{References}

[ 1 ] KIRSCHNER I N . Results of selected experiments involving supercavitating flows[M] . Defense Technical Information Center, 2001: 1-14 .

[ 2 ] YOUNG Y, KINNAS S. Numerical modeling of supercavitating propeller flows[J]. Journal of Ship Research , 2003 , 47(1): 48-62.

[ 3 ] VASIN A D. The principle of independence of the cavity sections expansion (Logvinovich principle) as the basis for investigation on cavitation flows[C]. VKI Special Course on Supercavitating Flows. Brussels: RTO-AVT and VKI, 2001: 105-131.

[ 4 ] SEMENENKO V N. Dynamic processes of supercavitat ion and computer simulation[C]. VKI Special Course on Supercavitating Flows. Brussels: RTO-AVT and VKI, 2001: 239-268.

[ 5 ] B. Milwitzky, Generalized Theory for Seaplane Impact[J]. NACA-TR-1103, 1952. 20-24.

[ 6 ] T. Kiceniuk, An experimental study of the hydrodynamic forces acting on a family of cavityproducing conical bodies of revolution inclined to the flow $[\mathrm{J}]$. Hydrodynamics Laboratory, California Institute of Technology, E-12.17, 1954. 40-46.

[ 7 ] P.R. Garabedian, Calculation of axially symmetric cavities and jets[J]. Pacific Journal of Mathematics. 4 (1956) 611-684.

[ 8 ] Robert K, Charles H, John C. Experimental study of ventilated cavities on dynamic test model[A]. Fourth International Symposium on Cavitation California Institue of Technology, 2001. Session B31004.

[ 9 ] S.S. Kulkarni, Studies in the dynamics of a supercavitating vehicle, M.E. Thesis, Department of Mechanical Engineering, Indian Institute of Science, 1998.5-10. 